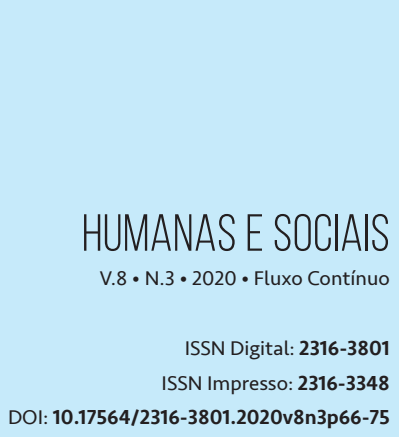

\section{PERFIL DE HOMENS QUE PROCURAM UM SERVIÇO DE SAÚDE NA REGIÃO SUL DO MUNICÍPIO DE SÃO PAULO'}

\section{PERFIL DE HOMBRES QUUE BUSCAN UN SERVIIIO DE SALUDEN LA REGIÓN SUR DEL MUNICIIIO DE SÃO PAULO}

PROFILE OF MEN LOOKING FOR A HEALTH SERVICE IN THE SOUTHERN REGION OF THE CITY OF SÃO PAULO

Sirlei Novato Maria Cecília Leite de Moraes ${ }^{3}$

\section{RESUMO}

O escopo da pesquisa foi conhecer o perfil sociodemográfico de homens que procuram um serviço de saúde na região Sul do município de São Paulo. Para tal, foi desenvolvida uma pesquisa de campo, exploratória e descritiva, de abordagem quantitativa, com uma amostra de conveniência formada por 51 indivíduos. A coleta de dados foi feita por meio de um questionário. Os resultados obtidos evidenciaram a presença de homens jovens (55\%), bran$\cos (43 \%)$, casados (65\%), com ensino médio (63\%) e católicos (44\%). Pôde-se observar sutis mudanças no autocuidado masculino, já que outros trabalhos mostram uma maior frequência de idosos, por problemas de saúde pré-existentes. Neste sentido, mesmo diante de um trabalho inicial, vislumbra-se uma mudança de cenário, a qual poderá ser chancelada por novas pesquisas sobre o tema.

\section{PALAVRAS-CHAVE}

Saúde do Homem. Saúde Pública. Cuidado de Saúde. Pesquisa Interdisciplinar.

1 Trabalho desenvolvido na pós-graduação em Saúde Pública do UNASP, pela discente Sirlei Novato, orientado pela Prof. Dra. Maria Cecília Leite de Moraes, no ano de 2018. 


\section{RESUMEN}

El objetivo de esta investigación fue conocer el perfil sociodemográfico de los hombres que buscan un servicio de salud en la región sur de la ciudad de São Paulo. Para este propósito, se ha desarrollado una investigación de campo, exploratorio y descriptivo con abordaje cuantitativo, con una muestra de conveniencia compuesta de 51 personas. La recogida de datos se realizó por medio de un cuestionario. Los resultados obtenidos demostraron la presencia de hombres jóvenes (55\%), blancos (43\%), casados (65\%), con estudios secundarios (63\%) y católicos (44\%). Pudimos observar los cambios sutiles en el autocuidado de los hombres, ya que otros estudios mostraron una mayor frecuencia de ancianos, debido a problemas de salud preexistentes. En este sentido, aunque delante de un trabajo inicial, hay un cambio de escenario, el cual puede ser examinado por nuevas investigaciones sobre el tema.

\section{PALABRAS CLAVE}

Salud de los hombres. Salud pública. Cuidado de la Salud. Investigación interdisciplinaria

\section{ABSTRACT}

The scope of the research was to know the sociodemographic profile of men seeking a health service in the southern region of the city of São Paulo. For this, a field research, exploratory and descriptive, of quantitative approach was developed, with a convenience sample of 51 individuals. Data were collected through a questionnaire. The results obtained evidenced the presence of young men (55\%), white (43\%), married (65\%), high school (63\%) and Catholic (44\%). Subtle changes in male self-care may be observed, since other studies show a greater frequency of elderly people due to preexisting health problems. In this sense, even in the face of an initial work, we can see a change of scenery, which may be supported by new ones on the theme.

\section{KEYWORDS}

Man's helth. Public health. Health care. Interdisciplinary research. 


\section{INTRODUÇ̧̃̃o}

A baixa procura por serviços de saúde entre a população masculina sofre influência de fatores culturais relacionados ao gênero (LEVORETO et al., 2014). Como consequência, crescem as taxas de mortalidade entre os homens, causadas, principalmente, por doenças crônicas e neoplásicas (ALBANO; BASÍLIO; NEVES, 2010; SCHWARZ, 2012). Em 2008, o Sistema de Informação de Mortalidade (DATASUS-SIM) realçou que os óbitos na população masculina foram maiores do que na feminina e que se concentraram, principalmente, na faixa etária entre 20 e 29 anos. No que se refere às neoplasias, os números de casos alcançaram 82,7 casos em cada 100 mil homens em comparação a 62,5 casos em cada 100 mil mulheres (FERREIRA, 2013).

A negação da dor e do sofrimento é uma realidade masculina, já que a vulnerabilidade é socialmente atribuída ao sexo oposto. Dessa forma, a afirmação da masculinidade encobre a autopercepção sobre o corpo, fazendo com que os homens desconheçam o seu estado de saúde e cuidem-se menos ou, simplesmente, não se cuidem (MACHIN et al., 2011). Tais condutas os induzem a procurar os serviços de saúde quando em estado crítico, além de estimular a automedicação e fomentar as ações curativas em detrimento das preventivas (SCHRAIBER et al., 2010).

Cabe destacar que o conhecimento insuficiente sobre os benefícios dos cuidados preventivos atinge, especialmente, os indivíduos de baixa renda (SILVA et al., 2010). Os estudos mostram que tais sujeitos evitam as unidades de saúde com medo da descoberta de doenças, de procedimentos invasivos e pela timidez de terem seus corpos expostos aos profissionais de saúde (BONITO; LANDÓ; COSTA, 2010).

0 acolhimento dado aos indivíduos do sexo masculino é pouco atrativo e não especializado, condição que, também, influencia a baixa procura dos homens por serviços de saúde (GOMES et al., 2011).

A Política Nacional de Atenção Integral à Saúde do Homem (PNAISH) foi criada em 2009 e representou uma importante inovação no campo da saúde. Os principais objetivos da nova estruturação foram trazer esta coletividade para tais cuidados de sua saúde e assinalar à população geral sobre a realidade singular masculina (MINISTÉRIO DA SAÚDE, 2008).

Estudo recente apontou tênues transformações no sistema de cuidado masculino, referindo que os homens estão monitorando a saúde, fato que realça um avanço (MORAES; OLIVEIRA; SILVA, 2017). A fim de compreender melhor estas questões foi elaborado um estudo para identificar o perfil sociodemográfico de homens que frequentam um serviço de saúde.

\section{MÉTODO}

Este artigo é parte da pesquisa "Conhecendo atitudes masculinas" (NOVATO, 2018) elaborada para a conclusão do curso de pós-graduação em Saúde Pública do Centro Universitário Adventista de São Paulo (UNASP). Tratou-se de um estudo de campo, com objetivos exploratório e descritivo e abordagem quanti-qualitativa.

A amostra de conveniência foi constituída por 51 indivíduos. As informações foram obtidas por meio de um questionário formulado para a pesquisa. 0 instrumental foi respondido pelo entrevistado ou pelo pesquisador, quando foi necessário. 
Para compor o perfil da amostra, foram coletados os seguintes dados: idade, estado civil, grau de instrução e religião. A pesquisa foi realizada nas dependências do Posto Avançado do Hospital Adventista de São Paulo (serviço ambulatorial), localizado no bairro do Capão Redondo, na região Sul da cidade de São Paulo (WIKIPEDIA, 2018). É um local periférico, constituído por indivíduos em situação de maior vulnerabilidade socioeconômica (PONCIANO, 2004).

Todos os participantes assinaram o Termo de Consentimento Livre e Esclarecido. A pesquisa aconteceu entre os meses de outubro e dezembro de 2017, após a aprovação do Comitê de Ética em Pesquisa (CEP) do Centro Universitário Adventista de São Paulo - UNASP, sob o número 2.381.684.

\section{RESULTADOS}

Os resultados referiram as faixas etárias, cor/raça, estado civil, grau de escolaridade e religião declarados pelos respondentes. A Tabela 1 mostra a distribuição de número e porcentagem dos dados coletados para conhecer o perfil sociodemográfico da população amostral. A idade dos entrevistados variou entre 18 e 81 anos. Quanto ao estado civil, grande parte se declarou casado (65\%). Além disso, um número importante de indivíduos (63\%) referiu possuir ensino médio completo.

Tabela 1 - Número e porcentagem de variáveis sociodemográficas coletados da população amostral

Variáveis

$\mathbf{N} \%$

Faixa Etária

$18-38$
$39-59$
$60-81$

$28(55 \%)$

$16(31 \%)$

$7(14 \%)$

\section{Cor/Raça}

\begin{tabular}{ll} 
Branca & $22(43 \%)$ \\
Parda & $21(41 \%)$ \\
Negra & $1(2 \%)$ \\
Não declarou & $7(14 \%)$ \\
\hline
\end{tabular}


Tabela 1 - Número e porcentagem de variáveis sociodemográficas coletados da população amostral (continuação)

Variáveis $\quad \mathrm{N} \%$

\section{Estado Civil}

Casado

$33(65 \%)$

Solteiro

União estável

$17(33 \%)$

$1(2 \%)$

\section{Grau de Escolaridade}

Analfabeto e fundamental incompleto

$4(8 \%)$

Fundamental completo

$8(15 \%)$

Médio completo

$32(63 \%)$

Superior completo

$6(12 \%)$

Pós-graduação

$1(2 \%)$

\section{Religião}

Católica

Evangélico/Protestante

Cristão

$22(43 \%)$

$17(33 \%)$

$5(10 \%)$

Sem religião

$7(14 \%)$

Fonte: Dados da pesquisa.

\section{DISCUSSÃO}

É possível observar que 55\% dos homens que procuraram os serviços estavam na faixa etária entre 18 e 38 anos, com apenas 14\% na faixa etária de idosos. O resultado está consonante com um estudo que aborda o uso dos serviços (LEVORETO et al., 2014). A procura de serviços por homens jovens pode pontuar uma mudança de atitude no que diz respeito ao cuidado. Por outro lado, uma menor busca dos serviços por parte da população idosa merece destaque, já que se espera que pessoas deste grupo tenham mais e maiores necessidades de atenção à saúde (VERAS, 2012).

Oitenta e quatro por cento dos homens que responderam à pesquisa se autodeclararam brancos e pardos, apenas $1 \%$ de indivíduos da amostra se intitularam como negro. 0 dado pode indicar constrangimento de se declarar negro, o que não é incomum pelo contexto social vigente, ao 
mesmo tempo em que pode apontar questões de acesso deste contingente aos serviços de saúde (SANTOS; NETO, 2011; ZAMORA, 2012). Ressalta-se que um número importante de indivíduos não forneceu a informação.

A pesquisa mostra, confirmando os dados existentes, uma aproximação entre o número de católicos e evangélicos (MACHADO, 2012). 0 aspecto religioso pode ser um fator de proteção, influenciando a maneira de sentir e agir e impactando a forma de se cuidar (SALGADO; ROCHA; CONTI, 2007; PANZINI; BANDEIRA, 2010). A informação sobre aspecto religioso é importante na medida em que constitui a integralidade e complexidade humana e dos indivíduos na sociedade.

Mais da metade dos respondentes (63\%) declarou ter concluído o ensino médio, número que fica muito acima dos dados da região Sudeste, na qual apenas $29,7 \%$ dos indivíduos possuem este grau de instrução. Ao mesmo tempo, o número de analfabetos é compatível com a média nacional, mas acima do esperado na região Sudeste, que apresenta índices de analfabetismo na casa dos 3,8\% (PRONI, 2017).

No que se refere ao estado civil, $65 \%$ da população amostral se declara casada. Acrescido o número que relatou união estável, o número alcança $67 \%$. 0 resultado é discordante com os achados que não encontram associação entre ser casado e cuidar da saúde (ALVES et al., 2011). Por outro lado, uma pesquisa revela que a mulher/companheira é um forte ator social no que diz respeito ao cuidado (GALASTRO; FONSECA, 2006). A existência desta parceria representa fator positivo no cuidado da saúde masculina.

\section{CONCLUSÕES}

A presença de homens jovens, frequentando serviço de saúde é uma pauta de grande relevância, apoia a ideia de mudança de atitude no sistema de cuidado masculino.

Apesar de um número importante de indivíduos terem omitido a informação quanto à cor, é possível destacar que não negros foram mais presentes no serviço de saúde, fato que retrata a realidade do país.

Ficou evidente a associação entre o grau de instrução e o autocuidado. Ainda, cabe enfatizar o nível de escolaridade entre os participantes do estudo, haja vista que a pesquisa aconteceu em local desfavorecido no âmbito socioeconômico.

Os dados obtidos permitem mencionar a importante relevância da presença de parceiro no contexto do cuidar. Dado este que, por vezes, tem se destacado no campo da saúde masculina.

Apesar do entendimento de que há muito para se conhecer sobre universo do cuidado masculino, é possível reconhecer mudanças na atualidade. A importância relaciona-se principalmente ao fato da pesquisa ter acontecido em serviço ambulatorial geral. Ressalta-se as condições socioambientais pouco favoráveis, depreende-se que as transformações atingem diferentes camadas da população e este é um grande dado. Claro está que serão necessários muitos outros estudos, mas boas novas já despontam no cenário. 


\section{REFERÊNCIAS}

ALBANO, Bruno Ramos; BASÍLIO, Marcio Chaves; NEVES, Jussara Bôtto. Desafios para a inclusão dos homens nos serviços de atenção primária à saúde. Revista Enfermagem Integrada, v. 3, n. 2, p. 554-63, 2010.

ALVES, Railda Fernandes et al. Gênero e saúde: o cuidar do homem em debate. Psicologia: Teoria e Prática, v. 13, n. 3, p. 152-66, 2011.

BONITO, Rosuita Fratari; LANDÓ, Leonardo; COSTA, Débora de Sousa Ribeiro. Discutindo saúde do homem em unidades básicas de saúde da família, em Uberlândia, MG. Em Extensão, Uberlândia, v. 9, n. 1, p. 163-72, 2010.

FERREIRA, M. C. Desafios da política de atenção à saúde do homem: análise das barreiras enfrentadas para sua consolidação. Revista Eletrônica Gestão \& Saúde, Brasília, v. 4, n. 1, p. 183347, 2013.

GALASTRO, Elizabeth Perez; FONSECA, Rosa Maria Godoy Serpa da. A identidade masculina e feminina na visão dos profissionais de saúde de um serviço de saúde reprodutiva. Revista Mineira de Enfermagem, v. 10, n. 1, p. 37-40, 2006.

GOMES, Romeu; MOREIRA, Martha Cristina Nunes; DO NASCIMENTO, Elaine Ferreira, REBELLO, Lúcia Emília Figueiredo de Sousa; COUTO, Márcia Thereza; SCHRAIBER, Lilia Blima. Os homens não vêm! Ausência e/ou invisibilidade masculina na atenção primária. Ciência \& Saúde Coletiva, v. 16, supl. 1, p. 983-92, 2011.

LEVORATO, Cleice Daiana; MELLO, Luana Marques; DA SILVA, Anderson Soares; NUNES, Altacílio Aparecido. Fatores associados à procura por serviços de saúde numa perspectiva relacional de gênero. Ciência \& Saúde Coletiva, v. 19, n. 4, p. 1263-74, 2014.

MACHADO, Maria das Dores Campos. Religião, cultura e política. Religião \& Sociedade, v. 32, n. 2, p. 29-56, 2012.

MACHIN, Rosana; COUTO, Márcia Thereza; DA SILVA, Geórgia Sibele Nogueira; SCHRAIBER, Lília Blima; GOMES, Romeu; FIGUEIREDO, Wagner dos Santos; VALENÇA, Otávio Augusto; PINHEIRO, Thiago Félix. Concepções de gênero, masculinidade e cuidados em saúde: estudo com profissionais de saúde da atenção primária. Ciência \& Saúde Coletiva, v. 16, n. 11, p. 4503-12, 2011. 
MINISTÉRIO DA SAÚDE. Secretaria de Atenção à Saúde Departamento de Ações Programáticas Estratégicas. Política Nacional de Atenção Integral à Saúde do Homem: princípios e diretrizes. Brasília: Ministério da Saúde, 2008. Disponível em: http://bvsms.saude.gov.br/bvs/publicacoes/ politica_nacional_atencao_homem.pdf. Acesso em: 21 jan. 2019.

MORAES, Maria Cecília Leite de; OLIVEIRA, Robson da Costa; SILVA, Maria de Jesus. Uma questão masculina: conhecendo possíveis entraves para a realização dos exames de detecção do câncer de próstata. Revista Medica Herediana, Lima (Peru), v. 28, n. 4, p. 230-35, 2017.

PANZINI, Raquel Gehrke; BANDEIRA, Denise Ruschel. Escala de coping religioso-espiritual (escala CRE): elaboração e validação de construto. Psicologia em Estudo, v. 10, n. 3, p. 507-16, 2010.

PONCIANO, Levino. São Paulo 450 Bairros, 450 anos. São Paulo: Ed. Senac, 2004.

PRONI, Marcelo Weishaupt. Observações sobre a questão social no Brasil. Carta Social e do Trabalho, n. 36, p. 1-21, 2017.

SALGADO, Ana Paula Alves; ROCHA, Ruth Mylius; CONTI, Claudio de Carvalho. 0 enfermeiro e a abordagem das questões religiosas. Revista de Enfermagem UERJ, v. 15, n. 2, p. 223-8, 2007. SANTOS, Marzo Vargas dos; NETO, Vicente Molina. Aprendendo a ser negro: a perspectiva dos estudantes. Cadernos de Pesquisa, v. 41, n. 143, p. 516-37, 2011.

SCHRAIBER, Lilia Blima; FIGUEIREDO, Wagner dos Santos; GOMES, Romeu; COUTO, Márcia Thereza; PINHEIRO, Thiago Félix; MACHIN, Rosana; DA SILVA, Geórgia Sibele Nogueira; VALENÇA, Otávio. Necessidades de saúde e masculinidades: atenção primária no cuidado aos homens. Cadernos de Saúde Pública, v. 26, n. 5, p. 961-70, 2010.

SCHWARZ, Eduardo; GOMES, Romeu; COUTO, Márcia Thereza; DE MOURA, Erly Catarina; CARVALHO, Sarah de Araújo; DA SILVA, Simione Fátima César. Política de saúde do homem. Revista Saúde Pública, v. 46, p. 108-116, 2012.

SILVA, Maria Enoia Dantas da Costa; ALVARENGA, Willyane de Andrade; SILVA, Simone Santos; SILVA BARBOSA, Liana Dantas da Costa; ROCHA, Silvana Santiago. Resistência do homem às ações de saúde: percepção de enfermeiras da estratégia saúde da família. Revista Interdisciplinar NOVAFAPI, Teresina, v. 3, n. 3, p. 21-25, jul.-set., 2010.

VERAS, Renato Peixoto. Experiências e tendências internacionais de modelos de cuidado para com o idoso. Ciência \& Saúde Coletiva, v. 17, n. 1, p. 231-38, 2012. 
WIKIPEDIA, A Enciclopédia Livre. Capão Redondo. Disponível em: https://pt.wikipedia.org/w/index. php?title=Cap\%C3\%A3o_Redondo\&oldid=52821754. Acesso em: 3 set. 2018.

ZAMORA, Maria Helena Rodrigues Navas. Desigualdade racial, racismo e seus efeitos. Fractal: Revista de Psicologia, v. 24, n. 3, p. 563-78, 2012. 


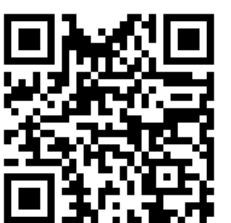

A autenticidade desse artigo pode ser conferida no site https://periodicos. set.edu.br
2 Enfermeira; Pós-Graduada em Saúde Pública pelo Centro Universitário Adventista de São Paulo - UNASP.

E-mail: maria.leite@unasp.edu.br

3 Doutora em Saúde Pública pela Faculdade de Saúde Pública da Universidade de São Paulo; Coordenadora do Curso de Pós-Graduação em Saúde e Professora do Programa de Mestrado Profissional em Promoção de Saúde Pública - Centro Universitário Adventista de São Paulo. E-mail: maria.leite@unasp.edu.br

\section{(). (1) (-)}

Este artigo é licenciado na modalidade acesso abertosob a Atribuição-Compartilhalgual CC BY-SA

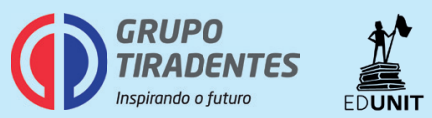

